\title{
Preschool Children Science Mental Representations: The Sound in Space
}

\author{
Konstantinos Ravanis ${ }^{1, *(\mathbb{D})}$, George Kaliampos ${ }^{2}$ (i) and Panagiotis Pantidos ${ }^{3}$ (D) \\ 1 Department of Educational Sciences and Early Childhood Education, University of Patras, \\ 26504 Rion-Patras, Greece \\ 2 Department of Education, School of Education, University of Nicosia, 2417 Nicosia, Cyprus; \\ kaliampos.g@unic.ac.cy \\ 3 Department of Early Childhood Education, National and Kapodistrian University of Athens, \\ 10680 Athens, Greece; ppantidos@ecd.uoa.gr \\ * Correspondence: ravanis@upatras.gr
}

Citation: Ravanis, K.; Kaliampos, G.; Pantidos, P. Preschool Children Science Mental Representations: The Sound in Space. Educ. Sci. 2021, 11, 242. https://doi.org/10.3390/ educsci11050242

Academic Editor: James Albright

Received: 21 April 2021

Accepted: 16 May 2021

Published: 18 May 2021

Publisher's Note: MDPI stays neutral with regard to jurisdictional claims in published maps and institutional affiliations.

Copyright: (c) 2021 by the authors. Licensee MDPI, Basel, Switzerland. This article is an open access article distributed under the terms and conditions of the Creative Commons Attribution (CC BY) license (https:// creativecommons.org/licenses/by/ $4.0 /)$.

\begin{abstract}
The aim of the current study was to examine the way in which preschool children deal with the concept of sound. For this purpose, a study was carried out in the context of detecting and categorizing the mental representations among young children of sounds which propagate through space from source to the receiver. Specifically, 91 preschool children aged 5-6 years voluntarily participated in individual semi-structured interviews which were carried out by three researchers in a special area of kindergartens. During these interviews, the children were asked to express their views on the three following axes: the concept of sound itself; the subjective characteristics of sound; and the phenomenon of the production and propagation of sound. The results of the research showed that while a small percentage of children recognized the propagation of sound in space, the vast majority of them associated sound with either the object that produced it or with the object that received it.
\end{abstract}

Keywords: genetic epistemology; mental representations; preschool children; physical sciences; sound

\section{Introduction}

The initiation of children aged 4-8 years into the world of Physical Sciences has been a growing interdisciplinary issue for education and research in recent decades. In a series of studies and teaching proposals that move toward diverse theoretical directions [1,2], a number of initiatives have been developed which over time have influenced international curricula and teaching strategies, and at the same time have contributed to the development of specific research and educational communities and traditions now recognized as Early Childhood Science Education. Along one of these directions, research orientations are placed within the broader context of Social Constructivism and Science Education, setting the study of young children's mental representations of concepts and phenomena of Physical Sciences in the core of the research process [3-5]. Within this framework exists the present study which investigated the mental representations of children for the propagation of sound in space.

\section{Theoretical Background and Literature Review}

\subsection{The Issue of Sound Comprehension}

The issue of a sound approach in education constitutes a systematic dimension of the curricula of Physics. Sound-both as a concept that developed through the history of human thought and as a fundamental term in science and education-has multiple scientific, cognitive and semiotic meanings [6]. Different disciplines investigate sound through different approaches and methodologies. In experimental psychology, research has shown that audio information can support visual information by creating a multimodal 
combined perception [7]. Auditory modalities improve the transfer of spatial information and spatial orientation performance and have a positive impact on the memory of spatial objects in a visual environment [8]. For example, in cartography, the auditory information of a property of an object in a map help memory in determining the location of the object. The integrated audiovisual information for the semantic property of a spatial object creates a binding between the object-identity and the object-location, which has an effect in memory process [9]. Although research in experimental psychology explores cognitive processes related to the connections between sound information and spatial entities as referring to the environment, the present study adopts the science education approach. According to physics, sound is an oscillation of molecules of an elastic medium (e.g., air) which, however, do not move from the source to the receiver. The source mechanically stimulates its neighboring molecules, which in turn stimulate those next to them, etc., resulting in a wave which finally stimulates the receiver's sensory organ. Physics deals with the construction of scientific/conceptual models that explore and describe this mechanism, while medicine, biology and neuroscience deal with the way the aforementioned mechanical stimulus which reaches the receiver in the form of a wave is encoded in terms of biological, cognitive processes, etc. Science education, among other things, deals with the possibility of a conceptual approach to these scientific models by students. From this perspective, the current study confines its interest in the way students think about sound.

Particularly in the education context, the basic characteristics of sound production and propagation have been approached in the earlier stages of curriculums that gradually lead to the conceptualization of sound as a wave phenomenon in the later stages. Students seem to encounter difficulties when they deal with specific aspects of sound, such as the way it is produced and it propagates through air, as well as its nature in general [10]. More specifically, they tend to attribute material properties to sound, advocating that since a 'solid' object cannot go through another one, it is impossible for sound to move across solids [11]. In addition, students often connect sound with its source, without making any reference to wave propagation [12]. Regarding sound production, students seem to focus on the context where it is generated [13]. The following common misconceptions are in line with this views that 'sounds can be produced without using any material objects', 'hitting an object harder changes the pitch of the sound produced', 'sounds can travel through empty space (a vacuum)', 'sounds cannot travel through liquids and solids', 'when waves interact with a solid surface, the waves are destroyed', etc. [14].

The materialistic nature of sound in students' thinking was studied by Eshach and Schwartz [15]. In particular, the researchers used the so-called 'substance schema' framework [16] in order to determine the extent to which eighth grade students attribute a range of material properties to sound (materialization of sound). Their results clearly showed that students tend to attribute pushable, frictional, containable and transitional features and properties to sound. All these are well reflected in the following students' statements: 'sound is pushed by the air, water, a barrier, is attracted by a stethoscope, or hits the walls'; 'sound experiences drag when it propagates through water'; 'sound (voice) is contained in bubbles'; and 'sound moves in straight, spiral or in a form of crescent shaped lines' [15] (pp. 746-753). Another finding of the Eshach and Schwartz [15] study suggested that students' thinking is governed by the view that the medium plays a key role in the propagation mechanism of sound. Along this line, students hold the view that sound tends to reformulate as it travels along diverse mediums.

Lautrey and Mazens [11] pointed out three naïve theories, comprising five mental models that encapsulate the substantial nature of sound. Regarding the first naïve theory, students exclusively attribute material substances to sound. Here, students claim that sound cannot move across solids as they are asked the question of how they are able to hear a sound that propagates through a material (model 1). Even in the case that they come across everyday life situations which disclose contradictory data, they tend to insist in their initial view. An exemplar paradigm of that is that they either refer to holes (model 2) or they perceive sound as a harder substance that the solid that can penetrate a wall (model 3) 
in order to explain the way they can hear noise from outside when they are in a closed room. According to the second naïve theory, students deal with sound as a substance, however, without attributing any kind of material properties to it. According to their view, sound can be invisible or transparent as a ghost or smoke (model 4). Finally, according to the third naïve theory, sound is regarded in a transmission context by adjacency where terms such as 'vibrating' or 'resonating' are used to describe its propagation mechanism. In line with this view are the findings of Hrepic, Zollman and Rebello [17], who demonstrated two main mental models: the wave model and entity model, as well as combinations of these. In particular, the wave model refers to sound as a vibration within a material medium which acts as a prerequisite for its propagation. The medium is made up of particles that oscillate around an equilibrium position. It is this oscillation that represents sound. On the other hand, according to the entity model, sound is an autonomous material entity that differs from the medium through which it propagates, which in turn becomes unnecessary in its propagation process. Thus, sound can propagate through empty space that exists between the particles of the medium, irrespectively of whether it affects them or not. In both cases, the propagation of sound is independent of the kinetic state of the particles that constitute the elastic medium.

\subsection{Sound Approach within Early Childhood Science Education}

At the pre-school level, the focus is both on the first organized sound experiences and the production and distinction of sounds as well as the initiation of children into musical sound. For example, during teaching activities, children classify, by association, sounds produced by various objects [18], while in other cases, they use the timbre (overtones) as a criterion for classifying the differences in materials, e.g., metallic sound for metals [19]. In addition, quite common is the usage of sounds as modalities, which along with oral speech and spatial entities compose narrative environments in the physical and/or digital space [20].

While for older children the main research issues seems to deal with the nature of sound, its wave character or its propagation through air and other materials [21,22], for younger children the most important issue has to do with the recognition of sound propagation in space. This difficulty constitutes a fundamental parameter for both the creation of learning and teaching activities as well as the orientation of relevant research, as it seems it goes beyond a simple mental representation and forms a prelogical feature of children's thinking, which requires systematic treatment. This aspect has been the subject of systematic research in the context of Genetic Epistemology. In a series of relevant studies, the hypothesis that was studied was whether children aged 7-8 years are able to conceptualize the propagation of non-tangible physical entities in space such as heat, light or sound [23,24]. This is due to the absence of a logical tool, operational transitivity, which allows the natural transition of such an entity between source and receiver to be understood as a two-step process: source $\rightarrow$ space and space $\rightarrow$ receiver. Children's thinking remains trapped in a direct source $\rightarrow$ receiver transition pattern and thus they do not attribute sound to its propagation any of its distinct characteristics [25,26]. Regarding the propagation of sound, for example, from one percussion supported in one box to another percussion, research has shown that '... Up to 7 years of age we only find ... immediate transmissions at a distance ... .... one diapason acts directly on the other because they are close (without touching each other) or similar, and without the box, the table or the air playing any role' [27] (pp. 214-215).

This structural barrier of children's thinking has been traced in a few studies of different theoretical orientation that have also tried to examine the way children 4-7 years old conceptualize sound. In most of these cases, young children associated sound with their own actions as well as with objects that produce sounds [12], while they considered that sound has the properties of a material object [13]. In accordance with the substantial entity of sounds are the findings of Gallegos-Cázares et al. [28]. In particular, the researchers studied the propagation of sound with 18 preschool children aged 4-5 years old and found 
that children of this age deal with sound as a material entity that requires space to propagate in order to be listened. Therefore, for example, when one speaks via a hose telephone, sound passes through the tiny hole and is received by the other's ear. Nevertheless, in the case in which the hole is folded and squeezed, sound gets stuck in it and cannot pass through. As a result, it cannot be heard. In this line of thought, if someone were to wear earmuffs, they could not listen as the substantial entity of sound cannot pass through the plastic material. Quite interesting is the fact that many children tried to attribute porosity (little holes) to that plastic material in order to match their premise with the fact that someone in reality can listen to a loud sound even when they wear earmuffs.

Kalogiannakis, Rekoumi and Chatzipapas [29], studied the mental representations of 4-6 year-old children and found that they were able to recognize the loudness and timbre of sounds, however, they showed greater difficulty with respect to the sounds of mechanical and musical instruments. They were also able to recognize the sounds that they had heard and were capable of matching the sounds to the corresponding images. From a perspective of the didactic treatment of relevant research findings, the authors proposed a series of didactic activities in the context of curriculum implementation. In this line, Smith and Trundle [30] proposed a 'sound study' unit that spans approximately one month and aspires to engage young children with the properties of sound through life, Earth and Physical Sciences. In particular, children have the opportunity via this unit to hear, see and feel sound through distinct activities. Therefore, for example, children experience a listening walk in the classroom, in the hallway and outside where they can hear different kinds of sounds. In addition, they visualize sound through the observation of a vibrating tuning fork, and they feel sound by placing their hands on their own throat while making various sounds (sing, talk, whisper, whistle). All these activities capitalize on children's interest and set the conditions for them to conceptualize sound in a way compatible with school-level Physics.

In the context of the Cultural-Historical Theory of Activity, having completed a preschool teacher training course, specific activities were developed with body sounds, sounds of familiar materials and objects of everyday life as well as the propagation of sound through various materials [31]. This research highlights the importance of a designed communication framework as well as the interactions and adherence to rules. Here, analysis is mainly focused in this direction and less on learning outcomes, which nevertheless seem satisfactory.

Research evidence through systematic interaction with 4-6-year-old children [32] showed the way that teachers, through a combination of play and learning, contribute to children's emerging scientific knowledge, which in turn enhances children's conceptual awareness about sound. Children interact with each other, as well as with their teacher by linking the contextual elements of the learning environment with a conceptual focus and understandings leading to the emergence of knowledge that sound is actually vibrations in a medium. Furthermore, acting in this way, their teachers contribute to bridge children's daily mental representations with the scientifically accepted concepts.

Christidou et al. [3] investigated 4.5-5.5 year-old children's noise awareness by recording ideas that referred to the conceptualization of noise, noise annoyance, health effects and protection measures. Regarding the conceptualization of noise, children define noise as annoyance, based on loudness, or identify it through examples. As for the source of noise, children display perceptions that attribute noise production to a human-made environment, natural environment, traffic, or work environment. Children's responses are also categorized in terms of subjectivity, as the same sound may be annoying to some children but not to others. In addition, it has been shown that activities based on socio-cognitive and multimodal teaching and learning processes can improve children's noise awareness to some extent.

Finally, in another study, representations of children aged 4-5 years about sound production, perception and propagation were diligently studied within the framework of embodied cognition [33]. The categorization of the research data revealed three levels 
of representations. In the first level, the sound is associated with the objects, while in the second, the sound is associated with the relation of objects to their environment, attributing to it some distinct, elementary features. In the third level, sound appears to have some inherent characteristics that increase its possibilities of interacting with objects and subjects of the environment. It seems that 'sound starts from perceptual elements and is linked to actions, although its complexity also shows that they incorporate characteristics that go beyond this corporal correlate' [33] (p. 952).

In the current research, an attempt was made to record mental representations of 5-6-year-old children about the recognition and propagation of sound in space, as well as about some basic features that characterize sound. The specific orientation of the research does not only lead to the recording of children's' representations, but also to a kind of classification which draws from the concept of operational transitivity.

Given that previous research has shown that children aged 5-6 years have the ability to express mental representations for non-tangible physical entities in space, before and/or after a teaching intervention, it seems that we can assume that children would also have the same cognitive ability for sound. Thus, the following three research questions (RQ) were posed:

(RQ1) How and to what extent do children identify sound as a physical entity as it appears in the context of everyday life.

(RQ2) How and to what extent do children differentiate sounds based on its subjective characteristics.

(RQ3) How and to what extent do children state that sound is produced and propagated as a distinct physical entity?

\section{Materials and Methods}

In the present study, a qualitative research approach was adopted [34].

\subsection{Participants}

This study involved 91 children from 5 years and 3 months to 6 years and 3 months (47 girls and 44 boys, average age: 5 years and 8 months) who attended 7 kindergarten classes in an urban area of Patras (Greece). These children, who volunteered to 'play' with the researchers, had no experience of organized sound activities in their classrooms. All socio-economic levels (low, middle, and high) were equally represented in the sample.

\subsection{The Procedure and the Interview}

The research was conducted through individual semi-structured interviews which took place in specially designed areas of kindergartens. The interviews lasted 12-17 min and were recorded, while at the same time protocols of non-verbal behaviors were observed. The Ethics Committee of the Department of Educational Sciences and Early Childhood Education of the University of Patras approved the study and consent procedures (the approval No: 12/8.3.2019).

The interview included tasks that covered all three research questions. Regarding the first research question, the mental representations of children about sound in everyday life (natural and artificial sounds, music) were investigated. With regard to the second research question, the issue of sound discrimination based on certain characteristics was investigated, while for the third research question, the effort was focused on establishing the recognition of the sound produced in a source and its propagation to the receiver. Regarding the third RQ, in task 3.3, the researchers decided to embed animated characters on Figure 1, so that children felt comfortable and happy. The usage of fictional characters did not affect the research results, as no kind of feature related to an imaginary world appeared in any of students' ideas. The aforementioned point had been also confirmed by an earlier pilot test for this image. In addition, the use of image (Figure 1) may have limited the expression of children's ideas, because it is a two-dimensional representation, as opposed to the fact that sound is evenly distributed throughout the three-dimensional 
space. This is a limitation of the research, which can be customized by designing data collection scenarios with 3D entities.

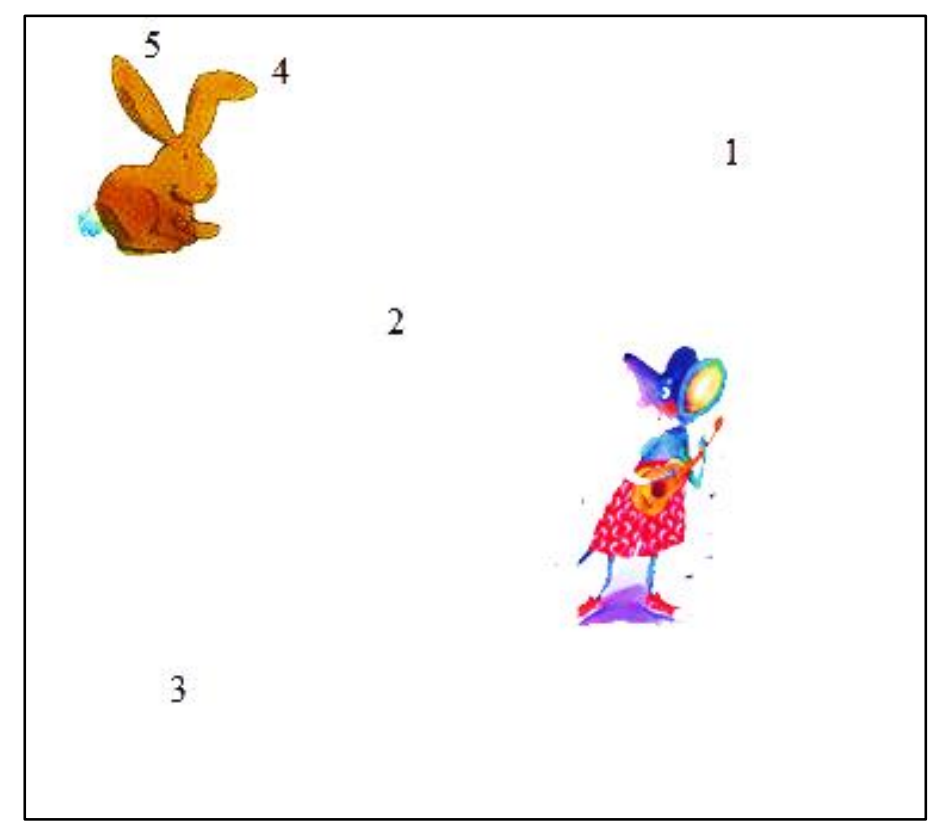

Figure 1. Which points does the singer's voice reach?

The interview questions and the order in which they were asked are presented in Appendix A (Table A1).

\subsection{Data Analysis}

Data analysis was based upon transcribed data of the discussions between children, the researcher and the individual observation protocols. The data were encoded and organized into 'categories'. Categories were derived in advance based on the ability of children to use transitional reasonings about sound and were modified by reference to the empirical data, i.e., whether they recognized that sound is propagated in space. As Cohen, Manion and Morrison [35] (p. 476) suggest 'content analysis involves coding, categorizing (creating meaningful categories into which the units of analysis-words, phrases, sentences etc.-can be placed), comparing (categories and making links between them), and concluding'.

Throughout interview questions, three levels of answers were distinguished that correspond to particular mental representations. At level a (sufficient answers), answers were classified in which the propagation of sound in space was fully recognized along with its own properties. At level $b$ (intermediate answers), answers were classified in which children's reasoning was dominated by both the sources and the receivers while the direct transition of sound between them was often recognized. At level c (insufficient answers), answers were classified in which the children's thinking was strongly tied to the voice as well as to the sources and receivers of sounds. The analysis and categorization of responses was performed by two independent researchers. The agreement among the two researchers was higher than $95 \%$.

\section{Results}

In what follows, the data of the interviews and children's performance to all tasks regarding the three research questions are presented. In particular, children's mental representations were categorized for each task and representative children's statements as well as frequencies and related frequencies of their answers are given below. 


\subsection{First Research Question}

Task 1.1. What is sound?

Regarding this question, three different types of mental representations were recorded, which stem from a spectrum of answers on one end of which lie the recognition of sound and on the other its direct connection with human voice.

a. Sound propagates in space. Here, children seemed to recognize that sound acts independently from both its point of production and the receiver. To quote pupil 6 (P. 6), 'It's something that reaches our ears .... . it comes from around' (Researcher. 'Why do you think this? How do you think it?'), 'Because we keep hearing it wherever we go...'.

b. Sound as a product of human and social activity. Answers in this category were focused on the source of the sound production. For example, P. 17. 'When we hit something $\ldots$ or on the roads from the cars... '.

c. Sound as a product of vocal expression. This category included answers that recognize an unambiguous relationship between sound and voice. While in some cases other types of sounds were mentioned, the main emphasis on sound production here remains voice. For example, P. 43. 'If we shout, the sound is generated', (R. 'Is there any other way for a sound to be produced?') 'With voices ... from mouths ... ', (R. 'Only human can produce sound?), 'Yes, humans ... and animals that bark let say ...'.

Table 1 presents the frequencies of children's responses to task 1.1.

Table 1. Children's responses to task 'what is sound?'.

\begin{tabular}{ccc}
\hline Categories of Responses & Frequencies & Relative Frequencies (\%) \\
\hline a. Sound propagates in space & 13 & 14.3 \\
\hline $\begin{array}{c}\text { b. Sound as a product of } \\
\text { human and social activity }\end{array}$ & 61 & 67 \\
\hline $\begin{array}{c}\text { c. Sound as a product of vocal } \\
\text { expression }\end{array}$ & 17 & 18.7 \\
\hline
\end{tabular}

Task 1.2. Could you tell me some kind of sounds that you know?

Children's answers to this question hold the same basic characteristics with those of task 1.1. Here, as well the way children express their views, the direction of their thinking is presented. Thus, from the analysis of their responses, the following three levels of answers were distinguished.

a. Sound propagates in space. Here, children seemed to attribute to sound the capability of propagation in space. For example, P. 33. 'The sound that comes out when a hammer strikes', (R. 'When you say 'it comes out' what do you mean?'), 'I mean that as the hammer strikes its target, a loud sound generates reaches our ears'.

b. Sounds as a product of human and social activity. In these answers, children focused their thinking on the actions that are capable of sound production. For example, P. 2. 'When two things strike, we hear the blow', (R. 'How do you think that? Could you give me an example?'), 'Yes ... when we clap ... our hands click ... '.

c. Sounds attributed exclusively to voices. Here, children seemed to limit their views about sound exclusively on voices. For example, P. 21. 'The voices of children at school', (R. 'Could you give me any other example?'), 'Yes, voices at home ... . or on the road'.

Table 2 presents the frequencies of children's responses to task 1.2. 
Table 2. Children's responses to task 'Could you tell me some kind of sounds that you know?'.

\begin{tabular}{ccc}
\hline Categories of Responses. & Frequencies & Relative Frequencies (\%) \\
\hline a. Sound propagates in space & 16 & 17.6 \\
\hline $\begin{array}{c}\text { b. Sound as a product of } \\
\text { human and social activity }\end{array}$ & 66 & 72.5 \\
\hline $\begin{array}{c}\text { c. Sound as a product of vocal } \\
\text { expression }\end{array}$ & 9 & 9.9 \\
\hline
\end{tabular}

Task 1.3. Is music a kind of sound?

Children were prompted to listen to an audio excerpt from an orchestral melody for a few minutes. They were then asked to answer the question of whether 'music is sound'. The researchers' goal here was to investigate whether children associated music, which is a particular cultural element, with other everyday sounds. From data analysis, the responses were classified into three distinct levels.

a. Music is sound. This category included answers that referred to the existence of music everywhere in space. For example, (R. 'Do you think that the music we listen is sound?') P. 64. 'Yes, it is ... but it does not look like other sounds ... it is somewhat special ... ', (R. 'So what is special about it?'), 'It is produced from instruments that play all together ... and individually sometimes'.

b. Music is associated with sound, in an unstable manner yet. In particular, when children focused their attention on sound perception, they did not seem to distinguish sound from music. However, when they referred to the sources of production, they seemed to recognize sound and music as different entities. For example, P. 60 'We hear both (music and sound) ... with our ears ... music is like sound. That is, it is like the sound we hear ... But they are made up differently ... ', (R. 'What do you mean they are 'made up' differently?'), 'Let's say ... the music from guitars and the sound from our voices ...'.

c. Music and sound totally differ. These answers record a clear distinction between 'sound' and 'music', i.e., music was understood as an element that was not related to sounds. For example, P. 51 'Music is 'music', it is not sound', (R. 'So, do they differ somewhere? What is different about them?'), 'Yes, of course. The music is songs ... That is, it is not done by ... when a voice is heard ... '.

Table 3 presents the frequencies of children's responses to task 1.3 .

Table 3. Responses to task 'Is music a kind of sound?'.

\begin{tabular}{ccc}
\hline Categories of Responses & Frequencies & Relative Frequencies (\%) \\
\hline a. Music is sound & 18 & 19.8 \\
\hline $\begin{array}{c}\text { b. Music is associated with sound, } \\
\text { in an unstable manner yet. }\end{array}$ & 56 & 61.5 \\
\hline c. Music and sound totally differ & 17 & 18.7 \\
\hline
\end{tabular}

\subsection{Second Research Question}

Task 2.1. Are all sounds the same? If not, where do they differ?

This question formed the initial basis for the development of dialogues in order to identify the subjective characteristics of sound that are important for young children. The following three categories of responses emerged in these discussions.

a. Answers in which differences are mainly identified on the different subjective characteristics of sound. Here, children's responses are mainly related to loudness and timbre while the distinction of sounds seems to be a constant element of their reasoning. For example, P. 46. 'They are not all the same. Others are strong and conflict pain to our 
ears, and other ... we do not even listen to them .... or let's say if we are far away we do not hear well when someone speaks', (R. 'Could we distinguish people who speak the same loud?'), 'Do you mean to distinguish who is it? Who is talking?' (R. 'Exactly to distinguish who speaks when two people speak the same loud'). 'Yes, it is quite easy ... my mom has a totally different voice from my sister Joana'.

b. Answers that recognize limited differences in sounds. This category included children's responses that pointed out differences between sounds in an instable and/or difficult manner. For example, P. 11. 'Hmmm ... it seems to me that they are not the same ... that is ... I do not know', (R. 'Do you want to explain it further? What do you think about these sounds that are not the same?'), 'I think they are the same but sometimes they do not look alike ... they differ', (R. 'Could you just tell me two of these sounds to make it clearer?'), 'The loud and the quiet sound'.

c. Answers in which no criteria were found for sound discrimination. Few children were not able to use criteria for sound discrimination. From the relevant discussions, it seemed that although these children recognized differences between sounds, they were not able to find indicators of sound discrimination. For example, P. 3 'They are the same ... they do not change ... ', (R. 'I mean if we hear two different sounds are they the same?'), 'They are the same', (R. 'So, does that mean that the voice of your mother and the sound that comes from TV are the same?) 'No, it's a totally different thing my mother voice and the sound from TV ... nevertheless, the sound does not change'.

Table 4 presents the frequencies of children's responses to task 2.1 .

Table 4. Responses to task 'Are all sounds the same? If not, where do they differ?'.

\begin{tabular}{ccc}
\hline Categories of Responses & Frequencies & Relative Frequencies (\%) \\
\hline $\begin{array}{c}\text { a. Differences based on subjective } \\
\text { characteristics of sound }\end{array}$ & 24 & 26.4 \\
\hline $\begin{array}{c}\text { b. Recognition of limited subjective } \\
\text { characteristics of sound }\end{array}$ & 53 & 58.2 \\
\hline $\begin{array}{c}\text { c. Absence of criteria for sound } \\
\text { discrimination }\end{array}$ & 14 & 15.4 \\
\hline
\end{tabular}

Task 2.2. Behind you there are some objects made of iron, wood and glass. I am going to hit two of them and I would like you to guess which one I am hitting each time. For example, if I hit these two objects made of plastic, they make that sound. Let us see if we can recognize the sounds without observing the objects that being hit.

With this task, an attempt was made to approach the familiarity of children with the timbre of sounds produced from ordinary everyday materials. At this stage, we did not use sounds such as the voices of children's parents, as the strong social marking creates an experiential relationship with the timbre of these sounds that goes far beyond the physical characteristics of the sound. In this task, we generated six sound stimuli by hitting two metal knives, two wooden spoons and two glass bottles in succession. Later, the stimuli were generated through hitting a metal with a wooden object, a wooden with a glass object and a glass object with a metal object.

The categorization of children's responses was based on whether the origin of the sound was recognized or not. For example, P. 77 'It's the irons', P.41 'The iron hit the wood', P. 1 'It's probably the knives' (two glass bottles collide with each other). It should be noted that sounds produced by objects of the same material were much more recognizable than those produced by objects of different materials. Moreover, children who failed to distinguish sounds often gave their response instantly. By so doing, instead of disclosing their failure, they gave the wrong impression that they did not realize that a kind of discrimination was possible. Thus, regarding the recognition of ordinary everyday sounds, children were classified into the following three categories: 
a. Those that satisfactorily recognized $5-6$ sounds;

b. Those that satisfactorily recognized $3-4$ sounds;

c. Those that either satisfactorily recognized 1-2 sounds or do not answer at all.

Table 5 presents frequencies of children's responses to task 2.2.

Table 5. Responses to task related to the recognition of sounds that come from everyday materials.

\begin{tabular}{lcc}
\hline \multicolumn{1}{c}{ Categories of Responses } & Frequencies & Relative Frequencies (\%) \\
\hline a. Recognition of $5-6$ sounds & 13 & 14.3 \\
\hline b. Recognition of 3-4 sounds & 38 & 41.7 \\
\hline c. Recognition of $1-2$ sounds & 40 & 44.0 \\
\hline
\end{tabular}

\subsection{Third Research Question}

Task 3.1. How are sounds produced, how are they made of?

With this question, an attempt was made to determine the way in which children approach the production of sound and especially whether they satisfactorily distinguish the concept of sound from the objects or the process of its production. As children do different centrations on sound creation, their answers fell into three distinct categories.

a. Answers in which sound is recognized as something that can be produced by any source. This category includes responses that refer to different audio sources, often without any kind of distinction between them. Essentially, the emphasis here is on the 'sound' itself. For example, P. 14. 'Sounds are produce all the time ... in many ways ... through voices, through objects ... the music, the cars ... ', (R. 'How can a sound be produced?'), 'Hmmm ... the objects have to hit each other to get out sound ... ' (R. 'How about cars, how does the sound come out?'), 'Hmmm ... the engine knocks ... even from the exhaust ... something hits there too ... and as the sound comes out we hear it'.

b. Answers in which limited natural and artificial sound sources are recognized while the distinction between objects of sound production and sound itself is blurred. Here, we have children's answers which refer to a limited number of audio sources and face difficulties in identifying different sources. For example, P. 28. 'Hmmm ... people's voices ... ', (R. 'Any other way?'), 'animals voices', (R. 'except of voices, is there any other way to produce a sound?'), 'hmmm ... . how else?', (R. 'I mean only from voices can produced sounds?'), 'Not only ... ', (R. 'Could you please express your thoughts?')?, 'hmmm ... let's say when a car passes ... ', (R. 'In any other way?').

c. Answers that focus exclusively on one type of voice source. In this category of responses, children focused on a single-signal type of sound source and mainly on the human voice. For example, P. 21. 'From the voices we make', (R. 'In any other way?'), 'When we talk ... when we cough ...' ', (R. 'Do you think of anything else ... of any other way that sounds can be produced?'), 'Oh yes, animals can also produce sounds', (R. 'How are they produced?'),' ... as the bark ... !'.

Table 6 presents frequencies of children's responses to task 3.1 .

Task 3.2. Where do the sounds go? If we produce a sound here on the table, inside the room, will it go somewhere else?

The answers to this question reflect the different mental representations that children use in order to approach the concept of sound. Having analyzed the data, the responses were classified into three distinct categories. 
Table 6. Responses to task 'How are sounds produced, how are they made of?'.

\begin{tabular}{ccc}
\hline Categories of Responses. & Frequencies & Relative Frequencies (\%) \\
\hline $\begin{array}{c}\text { a. Sound produced from a variety of } \\
\text { sources with a clear distinction between } \\
\text { sound and sources }\end{array}$ & 11 & 12.1 \\
\hline $\begin{array}{c}\text { b. Sound production from a limited } \\
\text { number of sources without a clear } \\
\text { distinction between sound and sources }\end{array}$ & 61 & 67 \\
\hline c. Correlation of sound mainly with voice & 19 & 20.9 \\
\hline
\end{tabular}

a. Answers where children recognize that sound is propagated everywhere in space. This category of responses is quite interesting as it highlights the ability of young children to recognize sound in space. For example, P. 39. 'Everywhere ... all around ... ', (R. 'How do you know that?'), 'Because, let's say, in case the bell of Saint Andreas rings, it can be also heard by us that we live by the sea .... All the neighbors can hear it .... It's like flying everywhere', and P. 64. 'Everywhere ... when it comes out of the CD let's say ... it goes everywhere. Everywhere ... like in concerts that everyone is able to listen to the music, irrespectively of the place it sits ... '.

b. Answers where sound is closely related to the way that is received by humans. In these responses, the receiver seems to hold a particularly important position, which leads to a kind of decentralization of children's thinking from the sources of sound production. For example, P. 10. 'In our ear', (R. 'How does this happen?'), 'It happens ... because we hear it ... ' and P. 88. 'Everywhere', (R. 'If we produce a sound here on the table, inside the room, will it go somewhere else?'), 'It will go around here', (R. 'Could you show me the exact point it will go?'), 'Yes ... here where we are sitting ... '.

c. Answers that focus on sound production. In these responses, the main focus stands on sound sources. For example, P. 30. 'They do not go anywhere ... ', (R. 'Could you explain it further to me?'), 'They are people's voices ... they do not go somewhere ... they just go along with peoples ... '.

Table 7 presents frequencies of children's responses to task 3.2.

Table 7. Responses to task 'Where does sound go?'.

\begin{tabular}{ccc}
\hline Categories of Responses & Frequencies & Relative Frequencies (\%) \\
\hline $\begin{array}{c}\text { a. Sound is propagated } \\
\text { everywhere in space }\end{array}$ & 14 & 15.4 \\
\hline $\begin{array}{c}\text { b. Focus on the perception of } \\
\text { sound by humans }\end{array}$ & 62 & 68.1 \\
\hline c. Focus on sound production & 15 & 16.5 \\
\hline
\end{tabular}

Task 3.3. Here, we can see a mouse who sings and a rabbit who wants to hear her songs. I will show you some points to tell me whether her voice could be heard there.

With this question, the children had the opportunity to choose points in space independently of the hypothetical listener. Therefore, by using this illustration, we sought to understand whether children are able to recognize the sound regardless of the source and the receiver.

Children's responses were classified into three distinct categories.

a. Answers where children recognize the propagation of sound throughout space. In this category of responses, all five points were selected without any special distinction. For example, P. 77. 'In all places ... here ... there ... everywhere', (R. 'How do you know that?'), 'Hmmm ... wherever we go we will listen'. 
b. Answers where sound is linked with an imaginary space defined by the linear source-receiver relationship. In these responses, the presence of sound was recognized in points 2, 4 and 5. For example, P. 10. 'There .... in the ears of the rabbit the song will be heard' and P. 70 (R. 'Somewhere else?'), 'Maybe here too (point 2) ... I guess ... ' (R. 'Why? How do you think this?'), 'Because if rabbit's ear placed there, I think it will be able to hear...' '.

c. Answers focused solely on the sound receiver. Some children's responses seem to focus solely on the sound receiver. For example, P. 52. 'In rabbit's place where he is able to hear ... here and there (points 4 and 5)' (R. 'Could you tell me how you think this?'), 'Hmmm ... it will be where he can hear ... that is in his ears'.

Table 8 presents frequencies of children's responses to task 3.3 .

Table 8. Responses to task 'Where do the sounds go?

\begin{tabular}{ccc}
\hline Categories of Responses & Frequencies & Relative Frequencies \% \\
\hline Sound propagates everywhere & 17 & 18.7 \\
\hline $\begin{array}{c}\text { Sound between the source } \\
\text { and the receiver }\end{array}$ & 60 & 65.9 \\
\hline Sound on the receiver & 14 & 15.4 \\
\hline
\end{tabular}

Table 9 presents the frequency of children's responses at each stage of the process, and the exact sequence of responses that each child provided during the interview.

Table 9. Frequencies (f) of pupils' representations $(n=91)$.

\begin{tabular}{|c|c|c|c|c|c|c|c|}
\hline & Answers & a. Sufficient & f & b. Intermediate & f & c. Insufficient & $\mathbf{f}$ \\
\hline \multirow{3}{*}{$\begin{array}{l}\text { First Research } \\
\text { Question }\end{array}$} & $\begin{array}{l}\text { Question } 1.1 \text {. } \\
\text { What is sound? }\end{array}$ & $\begin{array}{c}5,6,14,15,31,33,42,46,61 \\
69,77,84,91\end{array}$ & 13 & $\begin{array}{c}2,3,4,9,10,11,12,16,17,18,19,20 \\
22,23,24,25,26,27,29,32,34,35 \\
37,39,40,41,44,45,47,49,50,51 \\
52,54,55,56,57,58,59,60,63,64 \\
65,66,67,68,70,72,73,74,76,78 \\
79,80,81,82,83,85,87,88,90\end{array}$ & 61 & $\begin{array}{c}1,7,8,13,21,28,30,36 \\
38,43,48,53,62,71,75 \\
86,89\end{array}$ & 17 \\
\hline & $\begin{array}{l}\text { Question } 1.2 . \\
\text { Sound that are } \\
\text { recognized by } \\
\text { children. }\end{array}$ & $\begin{array}{l}5,6,12,14,15,31,33,42,46 \\
\quad 60,61,69,77,84,90,91\end{array}$ & 16 & $\begin{array}{c}2,3,4,8,9,10,11,13,16,17,18,19 \\
20,22,23,24,25,26,27,28,29,32, \\
34,35,36,37,38,39,40,41,44,45 \\
47,49,50,51,52,54,55,56,57,58 \\
59,62,63,64,65,66,67,68,70,72 \\
73,74,75,76,78,79,80,81,82,83 \\
85,87,88,89\end{array}$ & 66 & $\begin{array}{c}1,7,21,30,43,48,53,71 \\
86\end{array}$ & 9 \\
\hline & $\begin{array}{l}\text { Question } 1.3 . \text { Is } \\
\text { music a kind of } \\
\text { sound? }\end{array}$ & $\begin{array}{c}5,6,12,14,15,26,31,33,42 \\
46,55,61,64,69,77,84,90 \\
91\end{array}$ & 18 & $\begin{array}{c}2,3,4,8,9,10,11,16,17,18,19,21 \\
22,23,24,25,27,29,32,34,35,37 \\
39,40,41,44,45,47,49,50,52,54 \\
56,57,58,59,60,63,65,66,67,68 \\
70,72,73,74,76,78,79,80,81,82 \\
83,85,87,88\end{array}$ & 56 & $\begin{array}{c}1,7,13,20,28,30,36,38 \\
43,48,51,53,62,71,75 \\
86,89\end{array}$ & 17 \\
\hline \multirow{2}{*}{$\begin{array}{l}\text { Second Research } \\
\text { Question }\end{array}$} & $\begin{array}{l}\text { Question } 2.1 . \\
\text { Are all the } \\
\text { sounds the } \\
\text { same? }\end{array}$ & $\begin{array}{l}2,5,6,12,14,15,22,26,31 \\
33,40,42,46,55,58,60,61 \\
\quad 65,69,73,77,84,90,91\end{array}$ & 24 & $\begin{array}{c}4,8,9,10,11,13,16,17,18,19,20 \\
21,23,24,25,27,29,32,34,35,36 \\
37,39,41,44,45,47,49,50,51,52, \\
54,56,57,59,63,64,66,67,68,70 \\
72,74,76,78,79,80,81,82,83,85 \\
87,88\end{array}$ & 53 & $\begin{array}{c}1,3,7,28,30,38,43,48 \\
53,62,71,75,86,89\end{array}$ & 14 \\
\hline & $\begin{array}{l}\text { Question } 2.2 . \\
\text { Sound } \\
\text { recognition. }\end{array}$ & $\begin{array}{c}6,14,15,31,41,42,46,61,64 \\
69,77,84,91\end{array}$ & 13 & $\begin{array}{l}2,3,4,5,8,9,10,11,12,17,19,21 \\
22,24,26,27,28,32,33,34,35,37 \\
39,44,47,49,54,55,58,60,65,66 \\
\quad 73,81,82,85,89,90\end{array}$ & 38 & $\begin{array}{c}1,7,13,16,18,20,23,25 \\
29,30,36,38,40,43,45 \\
48,50,51,52,53,56,57 \\
59,62,63,67,68,70,71 \\
72,74,75,76,78,79,80 \\
83,86,87,88\end{array}$ & 40 \\
\hline
\end{tabular}


Table 9. Cont.

\begin{tabular}{|c|c|c|c|c|c|c|c|}
\hline & Answers & a. Sufficient & $f$ & b. Intermediate & $\mathrm{f}$ & c. Insufficient & $f$ \\
\hline \multirow{3}{*}{$\begin{array}{l}\text { Third Research } \\
\text { Question }\end{array}$} & $\begin{array}{l}\text { Question } 3.1 . \\
\text { How are sounds } \\
\text { produced? }\end{array}$ & $\begin{array}{c}6,14,15,31,42,46,61,69,77 \\
84,91\end{array}$ & 11 & $\begin{array}{c}5,3,4,8,9,10,11,12,16,17,18,19 \\
22,23,24,25,26,27,29,32,33,34 \\
35,37,39,41,44,45 \\
47,49,50,51,52,54,55,56,57,58 \\
59,60,62,64,65,66,67,68,70,72 \\
73,74,76,78,79,80,81,82,83,85 \\
87,88,90\end{array}$ & 61 & $\begin{array}{c}1,2,7,13,20,21,28,30 \\
36,38,40,43,48,53,63 \\
71,75,86,89\end{array}$ & 19 \\
\hline & $\begin{array}{l}\text { Question 3.2. } \\
\text { Where do } \\
\text { sounds go? }\end{array}$ & $\begin{array}{c}5,6,14,15,31,39,42,46,61 \\
64,69,77,84,91\end{array}$ & 14 & $\begin{array}{c}2,3,4,8,9,10,11,12,16,17,18,19 \\
20,21,22,23,24,25,26,27,28,29 \\
32,33,34,35,37,40,41,44,45,47 \\
49,50,51,52,54,55,56,57,59,60 \\
63,65,66,67,68,70,72,73,74,76 \\
78,79,80,81,82,83,85,87,88,90\end{array}$ & 62 & $\begin{array}{c}1,7,13,30,36,38,43,48 \\
53,58,62,71,75,86,89\end{array}$ & 15 \\
\hline & $\begin{array}{c}\text { Question } 3.3 . \\
\text { Sound in space. }\end{array}$ & $\begin{array}{c}5,6,12,14,15,31,33,42,46 \\
55,61,64,69,77,84,90,91\end{array}$ & 17 & $\begin{array}{c}2,4,8,9,10,11,13,16,17,18,19,20 \\
21,22,23,24,25,26,27,29,32,34 \\
35,36,37,38,39,40,41,44,45,47 \\
49,50,51,54,56,57,59,60,63,65 \\
66,67,68,70,72,73,74,76,78,79 \\
80,81,82,83,85,87,88,89\end{array}$ & 60 & $\begin{array}{c}1,3,7,28,30,43,48,52 \\
53,58,62,71,75,86\end{array}$ & 14 \\
\hline
\end{tabular}

\section{Discussion}

In the present study, mental representations of sound among 5-6-year-old children were studied. The focus of the study was not to record children's reflections on certain characteristics of sound such as noise [3], neither their aptitude of identifying everyday sounds and sound sources [31]. Instead, an attempt was made to explore children's ability on sound recognition in space. This choice is based on the interpretation given by Genetic Epistemology for the understanding of non-tangible concepts $[23,24,26,27]$ and is of double importance for the learning processes of young children. On the one hand, the determination of the approach of sound through mental representations that allow the transitional reasoning of two steps points out the possibility of young children to approach the existence of sound in space. On the other hand, this finding shows the direction in which teaching activities should be developed for children who do not adopt transitional reasonings. The findings of the present study indicate that preschool children are able to conceptualize to some extent that sound spreads everywhere in space and propagates from the source to the receiver. Undoubtedly, the form that sounds obtains through this propagation may be interpreted by the 'wave' and 'entity model' found by Hrepic, Zollman and Rebello [17]. Indeed, these models could be implied in children's responses regarding our three research questions. Nevertheless, as our study focused on 5-6-year-old pupils, in contrast to the sample studied by Hrepic et al. [17] which consisted of university students, we decided not to deal with the appearance of these models in our children's thinking.

In the three tasks of the first RQ, it seemed that $14-20 \%$ of students recognized sound independently of its sources of production, its origin, or particular forms that manifest itself such as musical sound. The conceptualization of sound as a distinct physical entity is a finding of relevant research that has focused on this issue [28,33]. However, the vast majority of children (61.5-72\%) refer to sound in close relation to the sources of production and the activities that produce distinct sounds, as well as to human hearing. This finding is consistent with similar studies that show the key role that the sources of sound play in the thinking of children aged 5-7 years $[12,29,31]$. Undoubtedly, the basic children's reasoning regarding the propagation of sound lies on the immediate transmission of an instantaneous stimulus. In addition, there is another group of children who seem to associate sound exclusively with voice $(9.9-18.7 \%)$. The attachment to the association of sound with voices creates a very limited centration of children's thinking which hinders any idea of sound as a physical entity. It should be noted that these children constantly distinguish sound from music.

Regarding the two tasks of the second $R Q$, an attempt was made to explore the subjective characteristic that is often attributed to sound by young children. Data analysis showed that children mainly refer to two basic subjective characteristics of sound: loudness and timbre. In particular, $14.3-19.8 \%$ of children satisfactorily distinguish these two 
characteristics and constantly express the mental representation of sound which own specific properties. A second category of children (58.2-61.5\%) hesitated to recognize differences in sounds, and when they do so, it is usually for sounds with which they are familiar, such as the voices of friends and family members. Finally, a relatively high percentage of children (15.4-44\%) seemed to face great difficulties in attributing subjective characteristics to sounds. This is compatible with the way they construct the concept of sound in their mind, as it is quite difficult for someone to attribute particular properties in something that is not recognized as a distinct phenomenon. These findings are consistent with similar previous studies where the production of sounds from different kind of materials has been explored $[18,19,31]$.

In the three tasks of the third $R Q$, an attempt was made to highlight the mental representations of children for the production and the distinction of sound from its sources. In all three tasks, a group of children (12.1-18.7\%) stated that sound can be produced in a number of different ways and propagates in space. A second group of children (65.9$68.1 \%$ ), however, seemed to refer in a consistent manner to the association between sound sources and the receiver, while very few of them were able to recognize the existence of sound between them. These were children who seemed to be on the verge of transition from immediate transmission to reasoning with characteristics of operational transitivity. The recognition of the presence of sound between a source and receiver is also a finding of another study [28]. Finally, a third group of children (15.4-20.9\%) insisted on the production of sound solely from voices and did not refer to any kind of propagation. A number of them seemed to recognize that apart from sources, voices only exist on receivers. These results are consistent with the findings of Piaget and Vergopoulo [27]. It should be noted, as mentioned above, that Eshach and Schwartz [15] stated that the way eighth-grade students conceptualize the notion of a wave or a medium affects the way they perceive both the nature of the sound and its propagation. Indicatively, a number of students who perceive sound as particles, draw different forms of particles in different media. In addition, students seem to attribute different mechanisms to the propagation of sound depending on the medium, for example, bubble-like in air, but ray-like in water (p. 759). In some cases, sound does not only seem to change form as it travels from one medium to another, but also the type of material through which is propagated is completely modified. However, given the fact that the present study did not make use of different mediums through which sound propagates, the effect of the medium on children's mental representations was not investigated. On the other hand, while the only medium that was available for discussion with children was air, it was apparent from relevant questions that children seem to recognize that sound is spread everywhere in space. Consequently, the construction of a mental transition step from the source to receiver seems likely to take place on children's thinking. Undoubtedly, it would be interesting in a future endeavor to investigate whether authentic ideas exist among preschool children related to sound propagation mechanisms.

Apart from dealing with the data of each research question separately, of particular interest was the finding of the stability of some children's reasoning which was expressed throughout all tasks. As shown in Table 9, eleven children $(6,14,15,31,42,46,61,69,77,84$, 91) seemed to recognize, along all tasks, the propagation of sound in space and in particular its propagation from sources to receivers. This finding is important because it underscores the ability of children, even from this age, to form in their minds a mental representation of sound based on a logical two-step thinking pattern for the propagation of invisible physical interactions in space. It is this mental representation that allows a significant prospect of children to be initiated to the world of sounds, as it provides a solid path for the development of teaching activities that will enable children of this age to deal with sounds and their basic characteristics. However, it would be quite interesting for feature research to investigate which kind of experiences and interactions enable such a developmental pathway which in turn leads some children to construct operational transitivity for sound. In this direction, a distinct stream of Early Childhood Science Education seeks to study 
the conditions and prerequisites for the construction of precursor models-meaning stable entities of thought which have characteristics compatible with those of school physics [36].

After all, as shown in Table 9, several children, apart from these eleven, often responded to a number of tasks with satisfactory responses, such as P. 5 scoring $6 / 8$ and P. 33, P. 12 and P. 90 scoring $4 / 8$ sufficient answers. Undoubtedly, the mind of these children was dominated by the mental representation of immediate transmission of sound and that is why their involvement with specialized teaching interventions is important. In general, the development of teaching activities needs special care and scaling as many children have a long mental path to go through until the conquest of transitivity. For instance, children P. 1, P. 7, P. 30, P. 43, P. 48, P. 53, P. 71, and P. 86, who gave insufficient answers to all tasks, need special teaching interventions to succeed in separating the concept of sound and voice, something that is not necessary for other children. However, in general, central elements of children's average and inadequate reasoning are closely related to the perceptual data of the proposed situations and especially to the materials that create sounds, the human voice or the actions that lead to the sound's creation. The transition to a state of reasoning that recognizes sound as a physical entity presupposes activities of decentralization from these elements.

\section{Conclusions}

In the present research, an attempt was made to investigate the mental representations of children aged 5-6 years for sound. Based on the theoretical framework of Genetic Epistemology, it seemed that conceptualizing sound, as is the case of heat [27] or light [26,37], requires the construction of a two-step transitional reasoning. While this construction is not expected to dominate children's thought at this age, it was shown to be possible. The research findings dictate the development of appropriate teaching activities which are based on the present study, in order to form mental representations compatible with those of school-level Physics. An axis of research in Early Childhood Science Education is now oriented in this direction.

Author Contributions: Conceptualization, K.R., G.K. and P.P; methodology, K.R., G.K. and P.P; software, K.R., G.K. and P.P; validation, K.R., G.K. and P.P; formal analysis, K.R., G.K. and P.P; investigation, K.R. and G.K.; resources, K.R., G.K. and P.P; data curation, K.R., G.K. and P.P; writingoriginal draft preparation, K.R., G.K. and P.P; writing-review and editing, K.R., G.K. and P.P; visualization, K.R., G.K. and P.P; project administration, K.R., G.K. and P.P. All authors have read and agreed to the published version of the manuscript.

Funding: This research received no external funding.

Institutional Review Board Statement: The study was conducted according to the guidelines of the Declaration of Helsinki, and approved by the Ethics Committee of Department of Educational Sciences and Early Childhood Education, University of Patras (protocol code No: 12/8.3.2019).

Informed Consent Statement: Informed consent was obtained from all subjects involved in the study.

Data Availability Statement: The data presented in this study are available on request from the corresponding author. The data are not publicly available due to privacy and ethical restrictions.

Acknowledgments: The authors would like to thank all of the participants who participated in this research.

Conflicts of Interest: The authors declare no conflict of interest.

\section{Appendix A}

In the appendix is presented Table A1 which shows the order of tasks in which they were presented to the children of the sample. 
Table A1. The order in which the tasks were presented to children.

\begin{tabular}{ccr}
\hline $\begin{array}{c}\text { Order } \\
1\end{array}$ & Number & Task \\
\hline 2 & 1.1 & What is sound? \\
\hline 3 & 1.2 & Could you tell me some kind of sounds that you know? \\
\hline 4 & 3.1 & Are all sounds the same? If not, where do they differ? \\
\hline 5 & 2.2 & $\begin{array}{r}\text { Behind you there are some objects made of iron, wood and glass. I am going to hit two of them and I would } \\
\text { like you to guess which one I am hitting each time. For example, if I hit these two objects made of plastic, } \\
\text { they make that sound. Let's see if we can recognize the sounds without observing the objects that being hit. }\end{array}$ \\
\hline 7 & 3.2. & Where do the sounds go? If we produce a sound here on the table, inside the room, will it go somewhere else? \\
\hline 8 & 1.3 & Is music a kind of sound? \\
\hline
\end{tabular}

\section{References}

1. Ravanis, K. Early Childhood Science Education: State of the art and perspectives. J. Balt. Sci. Educ. 2017, 16, 284-288.

2. Ravanis, K. The Physical Sciences in Early Childhood Education: Theoretical frameworks, strategies and activities. J. Phys. Confer. Ser. 2021, 1796, 012092. [CrossRef]

3. Christidou, V.; Dimitriou, A.; Barkas, N.; Papadopoulou, M.; Grammenos, S. Young noise researchers: An intervention to promote noise awareness in preschool children. J. Balt. Sci. Educ. 2015, 14, 569-585.

4. Kambouri, M. Children's misconceptions and the teaching of early years Science: A case study. J. Emerg. Sci. 2011, 2, 7-16.

5. Raviv, A.; Dadon, M. Teaching Astronomy in Kindergarten: Children's perceptions and projects. Ath. J. Educ. 2021, 8, 305-328.

6. Pantidos, P. Epistemic, cognitive and semiotic significations in science teaching: The case of sound. Europ. J. Educ. Stud. 2019, 6, 210-231.

7. Van der Burg, E.; Talsma, D.; Olivers, C.N.; Hickey, C.; Theeuwes, J. Early multisensory interactions affect the competition among multiple visual objects. Neuroimage 2011, 55, 1208-1218. [CrossRef] [PubMed]

8. Dinh, H.Q.; Walker, N.; Hodges, L.F.; Song, C.; Kobayashi, A. Evaluating the importance of multi-sensory input on memory and the sense of presence in virtual environments. In Proceedings of IEEE Virtual Reality Conference, Houston, TX, USA, 13-17 March 1999; IEEE Ed.: Houston, TX, USA, 1999; pp. 222-228.

9. Lammert-Siepmann, N.; Bestgen, A.K.; Edler, D.; Kuchinke, L.; Dickmann, F. Audiovisual communication of object-names improves the spatial accuracy of recalled object-locations in topographic maps. PLoS ONE 2017, 12, e0186065. [CrossRef]

10. Driver, R.; Squires, A.; Rushworth, P.; Wood-Robinson, V. Making Sense of Secondary School Science; Routledge: London, UK, 1994.

11. Lautrey, J.; Mazens, K. Is children's naïve knowledge consistent? A comparison of the concepts of sound and heat. Learn. Instr. 2004, 14, 399-423. [CrossRef]

12. Asoko, H.M.; Leach, J.; Scott, P.H. Classroom research as a basis for professional development of teachers: A study of students' understanding of sound. New prospects for teacher education in Europe II. In Proceedings of the 16th Annual Conference of ATEE, Amsterdam, The Neatherlans, 2-4 September 1992; Vonk, J.C., Giesbers, J.G.I., Peeters, J.J., Wubbles, T., Eds.; Universitaire Lerarenopleiding Vrije Universiteit: Amsterdam, The Neatherlans, 1992; pp. 109-119.

13. Watt, D.; Russel, T. Sound. Primary SPACE Project Research Report; University Press: Liverpool, UK, 1990.

14. Hapkiewicz, A. Naïve ideas in Earth science. MSTA J. 1999, 44, 26-30.

15. Eshach, H.; Schwartz, L.J. Sound stuff? Naïve materialism in middle-school students' conceptions of sound. Int. J. Sci. Educ. 2006, 28, 733-764. [CrossRef]

16. Reiner, M.; Slotta, J.D.; Chi, M.T.H.; Resnick, L.B. Naïve physics reasoning: A commitment to substance-based conceptions. Cogn. Instr. 2000, 18, 1-34. [CrossRef]

17. Hrepic, Z.; Zollman, D.; Rebello, N. Identifying students' mental models of sound propagation: The role of conceptual blending in understanding conceptual change. Phys. Rev. ST Phys. Educ. Res. 2010, 6, 020114. [CrossRef]

18. Krnel, D.; Watson, R.; Glazer, S.A. The development of the concept of "matter": A cross-age study of how children classify materials. Sci. Educ. 2003, 87, 621-639. [CrossRef]

19. Russell, K.; Longden, K.; McGuigan, L. SPACE Research Report: Materials; University Press: Liverpool, UK, 1991.

20. Lin, C.H. Application of a model for the integration of technology in kindergarten: An empirical investigation in Taiwan. Ear. Child. Educ. J. 2012, 40, 5-17. [CrossRef]

21. Eshach, H.; Lin, T.C.; Tsai, C.C. Misconception of sound and conceptual change: A cross sectional study on students' materialistic thinking of sound. J. Res. Sci. Teach. 2017, 5, 664-684. [CrossRef] 
22. West, E.; Wallin, A. Students' learning of a generalized theory of sound transmission from a teaching-learning sequence about sound, hearing and health. Int. J. Sci. Educ. 2013, 35, 980-1011. [CrossRef]

23. Piaget, J. Causalité et opérations. In Les Explications Causales; Piaget, J., Garcia, R., Eds.; PUF: Paris, France, $1971 ;$ pp. 11-140.

24. Piaget, J.; Lannoy, J.D. La Projection de la Lumière et de la Chaleur. In Recherche non Publiée sur la Causalité-Manuscrit Complet; Archives Jean Piaget: Geneva, Switzerland, 1967.

25. Ravanis, K. Représentations des élèves de l'école maternelle: Le concept de lumière. Int. J. Ear. Child. 1999, 31, 48-53. [CrossRef]

26. Ravanis, K.; Christidou, V.; Hatzinikita, V. Enhancing conceptual change in preschool children's representations of light: A socio-cognitive approach. Res. Sci. Educ. 2013, 43, 2257-2276. [CrossRef]

27. Piaget, J.; Vergopoulo, T. La transmission des vibrations entre deux diapasons. In La Transmission des Mouvements; Piaget, J., Ed.; PUF: Paris, France, 1972; pp. 213-227.

28. Gallegos-Cázares, L.; Flores-Camacho, F.; Calderón-Canales, E. Preschool Children's Reasoning about Sound from an InferentialRepresentational Approach. Educ. Sci. 2021, 11, 180. [CrossRef]

29. Kalogiannakis, M.; Rekoumi, C.; Chatzipapas, C. Playing on the journey of sound. A teaching proposal for children in early childhood. In Proceedings of the 10th International Conference on Computer Based Learning in Science CBLIS'2012, Learning Science in the Society of Computers, Barcelona, Spain, 26-29 June 2012; Pintó, R., López, V., Simarro, C., Eds.; Centre for Research in Science and Mathematics Education: Barcelona, Spain, 2012; pp. 279-285.

30. Smith, M.; Trundle, K. Shrieks and shrills: Investigating sound in preschool. Sci. Child. 2014, 52, 38-43.

31. Theodoraki, C.; Plakitsi, K. Analyzing activities in the course of science education, according to activity theory: The case of sound. US China Educ. Rev. A 2013, 3, 353-364.

32. Larsson, J. Contextual and conceptual intersubjectivity and opportunities for emergent Science knowledge about sound. Int. J. Ear. Child. 2013, 45, 101-122. [CrossRef]

33. Calderón-Canales, E.; Gallegos-Cázares, L.; Flores-Camacho, F. Sound representations in preschool students. J. Stud. Educ. Dev. 2019, 42, 952-999. [CrossRef]

34. Patton, M.Q. Qualitative Research and Evaluation Methods, 3rd ed.; Sage Publications: Thousand Oaks, CA, USA, 2002.

35. Cohen, L.; Manion, L.; Morrison, K. Research Methods in Education, 6th ed.; Routledge/Taylor \& Francis Group: Oxford, UK, 2007.

36. Ravanis, K. Mental representations of light propagation time for 10- and 14-year-old students: Didactical perspectives. J. Bal. Sci. Educ. 2019, 18, 276-285. [CrossRef]

37. Ravanis, K. Precursor models of the Physical Sciences in Early Childhood Education students' thinking. Sci. Educ. Res. Prax. 2020, $76,24-31$. 